\title{
Maternal cell-free DNA-based screening for fetal microdeletion and the importance of careful diagnostic follow-up
}

\author{
Svetlana A. Yatsenko, MD¹, David G. Peters, PhD', Devereux N. Saller, MD'1, Tianjiao Chu, PhD'1, \\ Michelle Clemens, MS, CGC' and Aleksandar Rajkovic, MD, PhD'
}

\begin{abstract}
Background: Noninvasive prenatal screening (NIPS) by next-generation sequencing of cell-free DNA (cfDNA) in maternal plasma is used to screen for common aneuploidies such as trisomy 21 in high risk pregnancies. NIPS can identify fetal genomic microdeletions; however, sensitivity and specificity have not been systematically evaluated. Commercial companies have begun to offer expanded panels including screening for common microdeletion syndromes such as 22q11.2 deletion (DiGeorge syndrome) without reporting the genomic coordinates or whether the deletion is maternal or fetal. Here we describe a phenotypically normal mother and fetus who tested positive for atypical $22 \mathrm{q}$ deletion via maternal plasma cfDNA testing.
\end{abstract}

Methods: We performed cfDNA sequencing on saved maternal plasma obtained at 11 weeks of gestation from a phenotypically normal woman with a singleton pregnancy whose earlier screening at a commercial laboratory was reported to be positive for a $22 \mathrm{q} 11.2$ microdeletion. Fluorescence in situ hybridization and chromosomal microarray diagnostic genetic tests were done postnatally.

Conclusion: NIPS detected a 22q microdeletion that, upon diagnostic workup, did not include the DiGeorge critical region. Diagnostic prenatal or postnatal testing with chromosomal microarray and appropriate parental studies to determine precise genomic coordinates and inheritance should follow a positive microdeletion NIPS result.

Genet Med advance online publication 8 January 2015

Key Words: cell-free DNA; DiGeorge; noninvasive prenatal screening; whole-genome sequencing
Noninvasive prenatal screening (NIPS) has become available over the past few years and has become increasingly requested in lieu of diagnostic procedures. Fetal aneuploidies such as trisomy 21 , as well as chromosomal microdeletions, can be identified by analyzing fetal cell-free DNA in the maternal plasma. ${ }^{1-3}$ For many pregnant women, the availability of NIPS offers a more accurate option over conventional prenatal methods for screening of fetal chromosomal abnormalities and removes the fear of miscarriage associated with invasive diagnostic testing. NIPS is currently offered exclusively through commercial entities. Some commercial companies have begun to offer microdeletion testing for common conditions such as DiGeorge syndrome. DiGeorge syndrome, or 22q11.2 deletion syndrome (OMIM 188400), affects 1 in 4,000 individuals and is associated with multiple anomalies including congenital heart disease, palatal defects, and immune deficiency. About $10 \%$ of 22q11.2 deletions are inherited from a mildly affected parent. DiGeorge syndrome in infants commonly requires acute management of hypocalcemia, surgery for conotruncal cardiac defects, and treatment of immunodeficiency. Therefore, prenatal detection plays an important role in pregnancy management and postnatal interventions. Here we present the clinical management and diagnostic testing initiated by a positive NIPS screen for 22q deletion.

\section{MATERIALS AND METHODS}

Our patient was enrolled in the NIPS research study at our institution as part of quality control, under an institutional review board-approved protocol. Maternal blood was obtained at 11 weeks of gestation and banked, independent of the blood sent to a commercial laboratory as part of clinical care. Cell-free DNA was extracted from maternal plasma and the whole-genome sequencing of the cell-free DNA was performed postnatally, as previously described. ${ }^{4}$ A peripheral blood sample was obtained from the patient's newborn child for confirmatory fluorescence in situ hybridization and microarray analyses. Fluorescence in situ hybridization was completed using a commercial TUPLE1 (HIRA) assay (Vysis/Abbott, Downers Grove, IL). A 180K comparative genomic hybridization plus single-nucleotide polymorphism platform (SurePrint G3 ISCA design; Agilent, Santa Clara, CA) was used for microarray analysis according to the manufacturer's protocol.

\section{RESULTS}

A 40-year-old gravida 6, para 2 underwent noninvasive cellfree DNA testing in her private physician's office because of advanced maternal age. In addition to the negative aneuploidy result, the test was reported as positive for a fetal DiGeorge

${ }^{1}$ Department of Obstetrics, Gynecology, and Reproductive Sciences, Magee-Womens Research Institute, University of Pittsburgh, Pittsburgh, Pennsylvania, USA. Correspondence: Aleksandar Rajkovic (rajkovic@upmc.edu) 
a

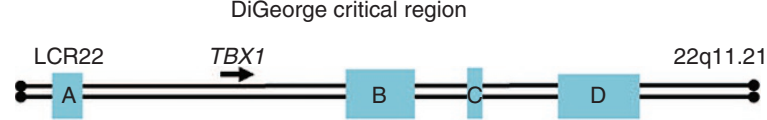

b

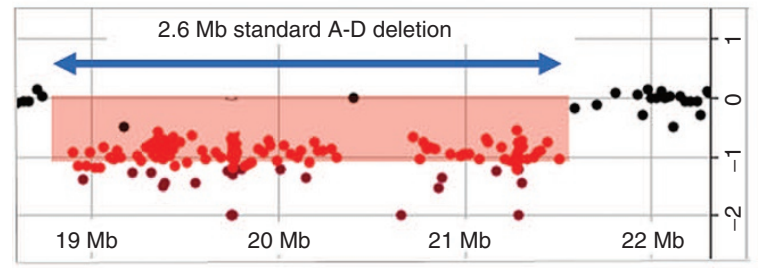

C

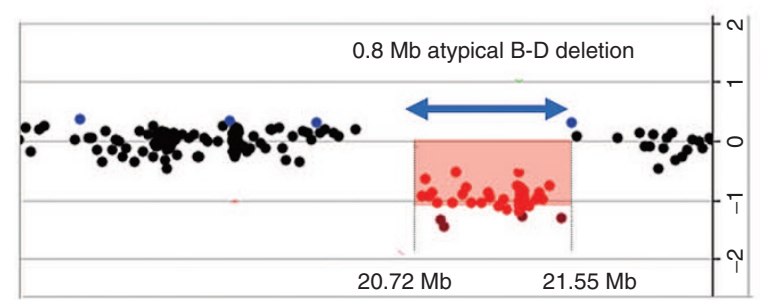

d

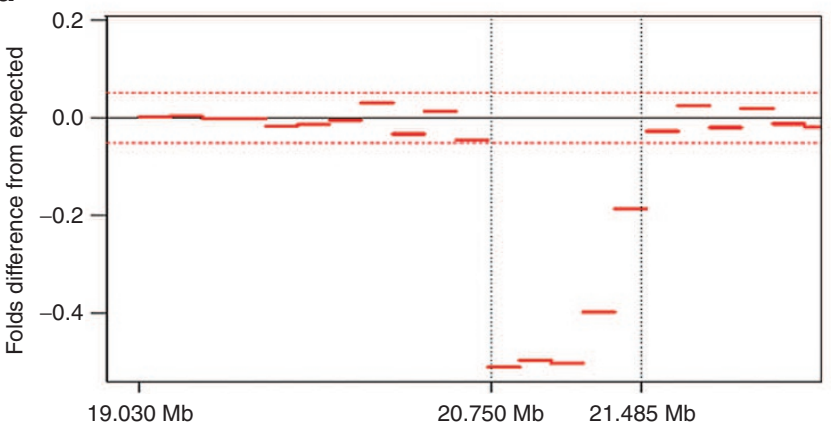

Figure 1 The results of microarray and NIPS testing. (a) Schematic presentation of the DiGeorge critical region on the proximal long arm of chromosome 22. The locations of the LCR22-A, LCR22-B, LCR22-C, and LCR22-D repeats are shown as blue boxes. The arrowhead represents the location and orientation of the TBX1 gene transcription. (b) The common 2.6- to 3-Mb deletion (found in $~ 85 \%$ of patients with DiGeorge/ velocardiofacial syndrome) occurs between the LCR22-A and LCR22-D repeats ( $A-D$ deletion) and includes $T B X 1$. A comparative genomic hybridization array plot from an individual with a standard A-D deletion is shown. Each dot represents an oligonucleotide probe; probes are arranged according to their physical map locations from the proximal (left) to the distal (right) long arm of chromosome 22 ( $x$-axis) and placed on a $\log _{2}$ scale ( $y$-axis). At the bottom, genomic coordinates (in megabases) are given according to the Human Genome Browser map (GRCh37/hg19). Black dots indicate DNA probes with normal copy number ( $\log _{2}$ is between +0.3 and -0.5 ). Red dots designate a chromosomal segment with a negative $\log _{2}$ ratio (less than -0.5), depicting the deletion region. (c) Array comparative genomic hybridization plot from the newborn baby showing an atypical deletion of the DiGeorge region with breakpoints between the LCR22-B and LCR22-D repeats (blue double arrowhead). The B-D deletion does not involve TBX1 and therefore cannot be detected by commercially available fluorescence in situ hybridization probes. (d) Maternal plasma at 11 weeks of gestation was used to isolate and perform whole-genome sequencing of cell-free DNA as described in ref. 1. The relative difference between the observed tag count in part of chromosome 22 in the patient's sample and the expected tag count for a normal sample was determined as described in ref. 4. The whole-genome sequencing of cell-free DNA shows a deletion between 20.75 and $21.485 \mathrm{Mb}$, which corresponds to the array comparative genomic hybridization findings. syndrome microdeletion. However, the report did not indicate genomic coordinates. The patient was counseled that the baby may be affected with DiGeorge syndrome and was referred to a tertiary-care center at 18 weeks of gestation for fetal echocardiography and ultrasound screens. Prenatal ultrasound demonstrated no apparent anomalies. The location and volume of fetal thymus and fetal echocardiogram were normal. Fetal growth restriction was detected in the third trimester and likely related to chronic hypertension. The patient declined diagnostic genetic testing during pregnancy. Because of the uncertainty of whether the NIPS result was a true or false positive, and the screening nature of the prenatal ultrasound, a recommendation to deliver at a tertiary-care center for a detailed postnatal examination was made. The mother developed severe preeclampsia and delivered by repeat cesarean delivery at 32 weeks, 5 days a 1,053-g boy with Apgar scores of 8 and 9. The baby was discharged to home at 5 weeks of age. Physical examination did not reveal craniofacial abnormalities and the postnatal echocardiogram did not identify heart defects. Because of the abnormal NIPS result, the DiGeorge critical region was evaluated by fluorescence in situ hybridization for a commonly deleted region (chr22:19,318,224-19,419,219). No deletion was detected in this region. A chromosomal microarray on the newborn showed a 790-kb microdeletion on chr22:20,719,112-21,505,417(hg19) (Figure 1a-c). This deletion involves the distal part of the classic DiGeorge region (B-D atypical), which does not include the TBX1 gene, thought to be responsible for the craniofacial features and heart malformations characteristic of DiGeorge syndrome. ${ }^{5}$ Microarray analysis of parental samples showed that the deletion was inherited from the mother, whose physical and echocardiographic examinations were unremarkable. Repeated whole-genome sequencing in our academic laboratory of maternal plasma DNA, obtained at 11 weeks gestation, discovered that coordinates of the deleted region match closely those found by microarray (Figure 1d).

\section{DISCUSSION}

NIPS is currently offered as an alternative to serum screening programs based on protein and hormone markers for high-risk pregnancies because of its superiority in detecting trisomy 21.,6${ }^{8}$ Moreover, societies such as the American College of Obstetrics and Gynecology support its use in high-risk patients. ${ }^{9}$ Despite some advantages, NIPS is a screening test. The recent addition of microdeletion detection involving 4p, 5p, 15q12, 22q11, and other regions marks an attempt by commercial companies to show the feasibility of identifying pathogenic microdeletions, as well as differentiating them from each other. Although NIPS can be used to uncover fetal microdeletions, positive results have been described in few cases. ${ }^{1-3}$ The accurate detection of microdeletions requires a higher number of sequencing reads, and its sensitivity and specificity are still unknown. An argument can be made that routine NIPS microdeletion screening at this time is premature and should be offered only under a 
research protocol until we understand its true sensitivity and specificity, and the technical and biological variables that affect the detection accuracy, as well as its relevance to phenotypic penetrance. Nonetheless, general clinicians have begun to offer deletion screening in addition to aneuploidy testing. The fetal fraction constitutes $10 \%$ or less of the maternal plasma, ${ }^{3}$ which makes determining whether the deletion is fetal or maternal, or both, difficult. Positive deletion testing using NIPS should be followed by diagnostic testing to confirm the fetal origin of the deletion, as well as by parental studies to establish inheritance and to provide accurate counseling for conditions with variable expressivity and incomplete penetrance. Moreover, in the absence of precise genomic coordinates, counseling about the phenotypic consequences of a particular genotype is difficult because deleted gene content is not reported. In our case the family lived in a rural area, $\sim 3 \mathrm{~h}$ away from the tertiary-care center. Because of the NIPS findings, our patient underwent multiple fetal ultrasound visits before her care was transferred to the tertiary-care center for closer monitoring, but this occurred at increased cost to the patient because of transportation, time spent, and delivery away from home. In our case both the patient and her child carried an atypical small 22q11.2 deletion that did not include the DiGeorge critical region. Deletions of this region have been associated with a variable phenotype and incomplete penetrance. The exact genomic coordinates of the abnormality would be extremely valuable for optimized prenatal management, accurate genetic counseling for our patient, and appropriate diagnostic postnatal testing. With an increased use of NIPS in prenatal diagnosis and the current shift to reporting microdeletions as well, having precise genomic coordinates is of great importance in order to determine affected gene content and to appropriately counsel patients. When NIPS detects a deletion, counseling the mother that she is at risk of having the deletion and performing diagnostic evaluation on the trio of the fetus, mother, and father for the origin of the genomic alteration are essential. NIPS, whether for identifying aneuploidy or copy-number variation, is a screening test and always warrants further counseling with an offer of additional diagnostic studies.

\section{DISCLOSURE}

The authors declare no conflict of interest.

\section{REFERENCES}

1. Peters D, Chu T, Yatsenko SA, et al. Noninvasive prenatal diagnosis of a fetal microdeletion syndrome. N Engl J Med 2011;365:1847-1848.

2. Srinivasan A, Bianchi DW, Huang H, Sehnert AJ, Rava RP. Noninvasive detection of fetal subchromosome abnormalities via deep sequencing of maternal plasma. Am J Hum Genet 2013;92:167-176.

3. Lau TK, Jiang FM, Stevenson RJ, et al. Secondary findings from non-invasive prenatal testing for common fetal aneuploidies by whole genome sequencing as a clinical service. Prenat Diagn 2013;33:602-608.

4. Chu T, Yeniterzi S, Rajkovic A, et al. High resolution non-invasive detection of a fetal microdeletion using the GCREM algorithm. Prenat Diagn 2014;34: 469-477.

5. Garcia-Miñaur S, Fantes J, Murray RS, et al. A novel atypical 22q11.2 distal deletion in father and son. J Med Genet 2002;39:E62.

6. McCullough RM, Almasri EA, Guan X, et al. Non-invasive prenatal chromosomal aneuploidy testing-clinical experience: 100,000 clinical samples. PLoS One 2014;9:e109173.

7. Palomaki GE, Kloza EM, Lambert-Messerlian GM, et al. DNA sequencing of maternal plasma to detect Down syndrome: an international clinical validation study. Genet Med 2011;13:913-920.

8. Norton ME, Brar H, Weiss J, et al. Non-Invasive Chromosomal Evaluation (NICE) Study: results of a multicenter prospective cohort study for detection of fetal trisomy 21 and trisomy 18. Am J Obstet Gyneco/2012;207:137.e1-137.e8.

9. The American College of Obstetricians and Gynecologists Committee on Genetics; The Society for Maternal-Fetal Medicine Publications Committee. Committee Opinion No. 545. Noninvasive prenatal testing for fetal aneuploidy. Obstet Gyneco/ 2012;120:1532-1534. 